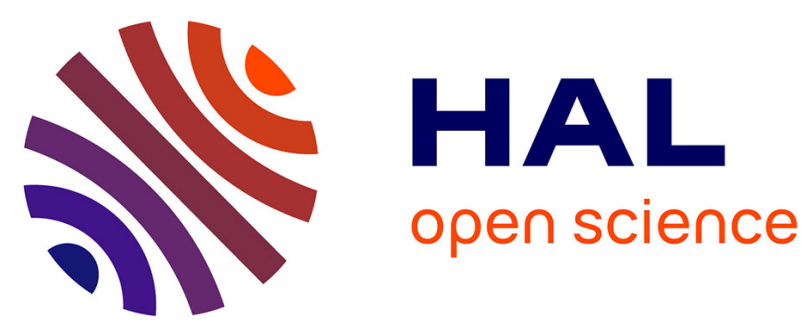

\title{
High loop rate adaptive optics flood illumination ophthalmoscope with structured illumination capability
} Elena Gofas-Salas, Pedro Mecê, Cyril Petit, Jessica Jarosz, Laurent Mugnier, Aurélie Montmerle Bonnefois, Kate Grieve, José-Alain Sahel, Michel Paques, Serge Meimon

\section{To cite this version:}

Elena Gofas-Salas, Pedro Mecê, Cyril Petit, Jessica Jarosz, Laurent Mugnier, et al.. High loop rate adaptive optics flood illumination ophthalmoscope with structured illumination capability. Applied optics, 2018, 57 (20), pp.5635-5642. 10.1364/AO.57.005635 . hal-01832999

\section{HAL Id: hal-01832999 \\ https://hal.science/hal-01832999}

Submitted on 9 Jul 2018

HAL is a multi-disciplinary open access archive for the deposit and dissemination of scientific research documents, whether they are published or not. The documents may come from teaching and research institutions in France or abroad, or from public or private research centers.
L'archive ouverte pluridisciplinaire HAL, est destinée au dépôt et à la diffusion de documents scientifiques de niveau recherche, publiés ou non, émanant des établissements d'enseignement et de recherche français ou étrangers, des laboratoires publics ou privés. 


\title{
High loop rate Adaptive Optics Flood Illumination Ophthalmoscope with structured illumination capability
}

\author{
Elena Gofas-Salas ${ }^{1,2,6,+}$, Pedro Mecế1,3,6,†, Cyril Petit ${ }^{1,6}$, Jessica Jarosz $^{1,3}$, Laurent \\ M. Mugnier ${ }^{1}$, Aurélie Montmerle Bonnefois ${ }^{1}$, Kate Grieve ${ }^{2,4,6}$, José Sahel ${ }^{2,4,5}$, Michel \\ PAQUes $^{2,4,6}$, ANd Serge MeImon ${ }^{1,6, *}$ \\ ${ }^{1}$ DOTA, ONERA, Université Paris Saclay, F-91123 Palaiseau - France \\ ${ }^{2}$ Institut de la Vision, Sorbonne Universités, UPMC Univ Paris 06, INSERM, CNRS, 17 rue Moreau, 75012 Paris, France \\ ${ }^{3}$ Quantel Médical, Cournon d'Auvergne, France \\ ${ }^{4}$ CIC 1423, INSERM, Quinze-Vingts Hospital, Paris, France \\ ${ }^{5}$ Department of Ophthalmology, The University of Pittsburgh School of Medicine, Pittsburgh, PA, United States \\ ${ }^{6}$ PARIS group - Paris Adaptive-optics for Retinal Imaging and Surgery, Paris, France \\ ${ }^{\dagger}$ These authors contributed equally to this work \\ *Corresponding author: serge.meimon@onera.fr \\ Compiled July 6, 2018
}

\begin{abstract}
The design and performance of an Adaptive Optics Flood Illumination Ophthalmoscope (AO-FIO) platform, based on eye motion and dynamic aberrations experimental analysis, is described. The system incorporates a custom-built Real Time Controler, enabling up to $70 \mathrm{~Hz}$ loop rate without jitter, and an $\mathrm{AO}-$ corrected illumination capable of projecting high resolution features in the retina. Wide-field $\left(2.7^{\circ} \times 5.4^{\circ}\right)$ and distortionless images from vessel walls, capillaries and the lamina cribrosa are obtained with an enhanced contrast and signal-to-noise ratio, thanks to the careful control of AO parameters. The high spatial and temporal resolution (image acquisition up to $200 \mathrm{~Hz}$ ) performance achieved by this platform enables the visualization of vessels deformation and blood flow. This system opens up the prospect of a return to favor of Flood Illumination Adaptive Optics systems provided that its high pixel rate and structured illumination capabilities are exploited. () 2018 Optical Society of America
\end{abstract}

OCIS codes: (170.4470) Ophthalmology; (330.4460) Ophthalmic optics and devices; (110.1080) Active or adaptive optics; (170.3880) Medical and biological imaging;(170.2655) Functional monitoring and imaging;

http://dx.doi.org/10.1364/ao.XX.XXXXXX

\section{INTRODUCTION}

Adaptive Optics (AO) can provide real-time correction of the wavefront aberrations introduced by the human eye, thus enabling retinal imaging with enhanced contrast and resolution [1-5]. The first AO corrected retinal images were obtained in 1997 [1] using a flood illumination technique (AO-FIO) recording en-face images with a camera. This seminal work gave rise to several similar systems, leading to Imagine Eyes' launch of its commercial version of AO-FIO (RTX1 [6]) based on the most popular technology at the time. Since then, AO-FIO has been replaced by AO scanning laser ophthalmoscope (AO-SLO) [5], i.e. the adaptation of the confocal scanning microscope to AOcorrected retinal imaging, due to improved optical sectioning and resolution. Evolutions proposed in the scanning microscopy field [7](dark field imaging, multiphotonic imaging, fiber optics components) have consequently been translated to ophthalmology on AO-SLO setups.

On the other hand, only a few research teams (see for instance recent research on neurovascular coupling $[8,9])$ developed custom-made AO-FIO systems, and most of published AO-FIO results are obtained with a commercial system. Although AOFIO holds the inherent advantage of distortionless high-speed imaging (while AO-SLO systems suffer from distortion artifacts in the presence of retinal motion, which hinders a reliable topography of retinal structures, e.g. small caliber vessels), it has not evolved significantly nor found inspiration in breakthroughs from full-field microscopy variants, such as structured illumination [10]. This technique, that requires the projection of high spatial frequency structures onto the retina, could bridge the gap between AO-FIO and AO-SLO in terms of optical sectioning and lateral and axial resolution [11, 12]. Moreover, increasing the 
adaptive optics loop rate, which has been shown to bring image quality enhancement in a scanning ophthalmoscope [13], has never been tried in $\mathrm{AO}-\mathrm{FIO}$, even though we have evidence that getting the $\mathrm{AO}$ loop rate from $10 \mathrm{~Hz}$ to $50 \mathrm{~Hz}$ is highly beneficial [14]. We present here details on an original AO-FIO platform featuring an $\mathrm{AO}$ loop rate reaching 50 to $70 \mathrm{~Hz}$ (depending on the configuration) and able to project $\mathrm{AO}$-corrected illumination patterns in the retina.

\section{METHODS}

The system consists of two optical subsystems, the Wavefront (WF) Sensing and Control subsystem and the Illumination and Detection subsystem (see Figure 1), which are detailed hereafter.

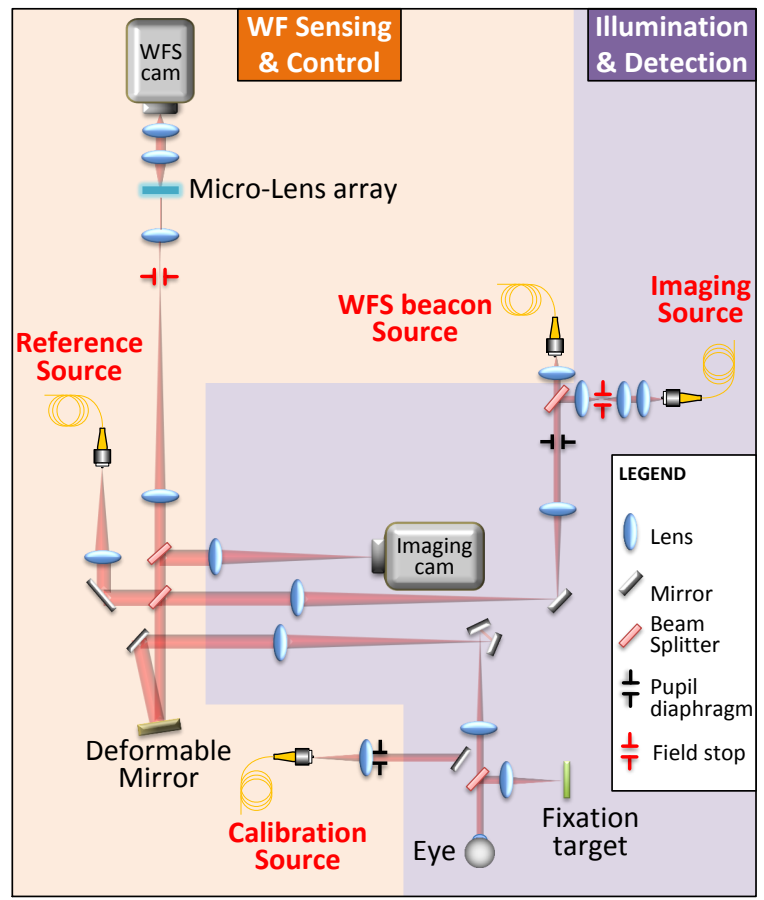

Fig. 1. Schematic drawing of the experimental set-up. The system can be divided into two subsystems. Wavefront (WF) Sensing \& Control, in yellow, is composed of a Reference Source (Ref Source), a Wavefront Sensor (WFS) (Micro-lens array, relay optics, and WFS camera), a WFS beacon source and a Deformable Mirror (DM). An additional calibration source can be inserted in place of the eye to calibrate the adaptive optics loop. The other subsystem, in purple, is Illumination \& Detection and is composed of the retinal imaging camera and the corresponding wide field imaging source.

\section{A. Wavefront sensing and control}

The wavefront sensing and control subsystem comprises three sources, a Wavefront sensor (WFS), and a deformable mirror (DM); it is operated via a Real Time Controller (RTC).

Wavefront sensing is performed using a custom-built Hartmann-shack (SH) sensor consisting of $30 \times 30$ microlens array. Relay optics conjugate the focal plane of the microlenses onto a Complementary Metal Oxide Semiconductor (CMOS) camera PCO.Edge 4.2 (PCO, Germany), where $14 \times 14$ pixels are allocated to each lenslet[15]. The microlens array pitch corresponds to $381 \mu \mathrm{m}$ in the eye pupil plane.

Wavefront correction is performed by a magnetic deformable mirror (ALPAO, France) with 97 actuators. A diaphragm limits the pupil size to $10 \mathrm{~mm}$ diameter, corresponding to $5 \mathrm{~mm}$ in the eye pupil plane.

A fibered Super Luminescent Diode (SLD) (Omicron, Germany) with nominal center wavelength of 750nm can be connected to three fiber mounts on the bench, corresponding to a reference source, on which reference wavefront sensor slopes are recorded; a calibration source, used to acquire the interaction matrix between the deformable mirror actuator voltages and the wavefront sensor slope measurements; a wavefront sensing beacon source used to project a point source beacon on the retina. The size of the beacon at the eye pupil is $4.5 \mathrm{~mm}$, with a $3 \mathrm{~mm}$ central obscuration to prevent stray light. The wavefront sensing beacon source is positioned upstream of the deformable mirror so that it benefits from Adaptive Optics correction. This layout also enables us to use the image of the wavefront sensor beacon as an in-situ Point Spread Function measurement. This Point Spread Function is not the same as the imaging one because the pupil size is not the same $(4.5 \mathrm{~mm}$ with a $3 \mathrm{~mm}$ central obscuration, whereas in the imaging pathway the pupil is set by the Deformable Mirror to a $5 \mathrm{~mm}$ diameter at the eye), but still allows a real-time assessment of the adaptive optics loop performance.

A custom-built Real Time Controler (RTC) comprising core libraries written in IDL (Exelis Visual Information Solutions, Inc., Boulder, Colorado, U.S.) within a LabView (National Instruments, Texas, U.S.) framework controls the adaptive optics. This solution enables us to tune control parameters and wavefront sensing methods with an interpreted coding language while enabling up to $70 \mathrm{~Hz}$ loop rate without jitter. Control law is an integrator with a 0.5 gain (ensuring a $45^{\circ}$ stability margin [16]), assuming a 2-frame delay (see chronogram in Fig. 2).

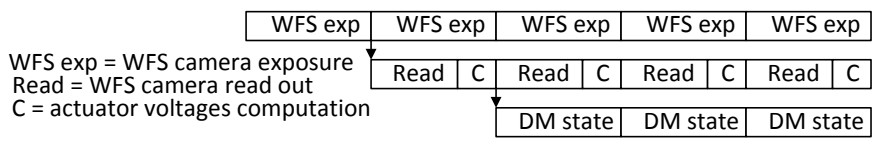

Fig. 2. Chronogram of the adaptive optics loop. Two frames (i.e. Wavefront sensor camera exposure time) separate a Wavefront sensor exposure and the corresponding Deformable Mirror (deformable mirror) state (2-frame delay loop).

\section{B. Illumination and detection}

The retinal imaging source is a collimated superluminescent diode (SLD) (Omicron, Germany) with a central wavelength of $860 \mathrm{~nm}(+/-50 \mathrm{~nm})$. The $2 \mathrm{~mm}$ diameter fiber tip of the illumination source is conjugated to the retina resulting in a $3.6^{\circ}$ diameter illumination field of view. The illumination beam passes through the deformable mirror before reaching the eye. This aberration compensation on the forward path enables the projection of high spatial frequency illumination patterns such as fringes onto the retina.

This light is then back-scattered by the retina towards a fast Scientific CMOS camera (Retinal imaging camera in Fig.1). The camera is an ORCA flash4-V2 (Hamamatsu, Japan), enabling $2048 \times 1024$ pixel frame acquisition at $200 \mathrm{~Hz}$, corresponding to more than $1.5 \mathrm{~mm} \times 0.75 \mathrm{~mm}$ field-of-view (approximately 
$\left.5.4^{\circ} \times 2.7^{\circ}\right)$. The magnification between the camera and the retina is such that each $6.5 \mu \mathrm{m}$ pixel corresponds to $0.73 \mu \mathrm{m}$ in the retina.

The camera is mounted on a motorized stage (Thorlabs, Germany). By moving the stage, we can focus on various layers of the retina.

\section{Subjects}

Acquisitions on three healthy subjects (two men, SM,MP; one woman, EG), have been performed on the AO-FIO. Every participation followed institutional guidelines and adhered to the tenets of the Declaration of Helsinki. Informed consent was obtained from subjects after the nature and possible outcomes of the study were explained. Subjects were seated in front of the FIO and stabilized with a chin and forehead rest. They were asked to stare at the fixation target, which is an image of a blue crosshair with a moving dot, enabling us to guide the subject's line of sight and explore various areas of the retina. Image acquisition sessions were performed in standard conditions with neither pupil dilation nor cycloplegia, in a dark room, leading to the largest accessible natural pupil dilation. The total light energy entering the eye from the illumination source and the WFS source are respectively under $400 \mu \mathrm{W}$ and $2.8 \mu \mathrm{W}$, which is less than half the power stipulated by the ocular safety limits established by ISO standards for group 1 devices.

\section{Real-time processing during acquisition}

During acquisition, a custom-made real-time image processing software is used to display retinal images with enhanced contrast. Each raw image is corrected for uneven background illumination, as brightness falls off towards the edges of the illumination patch. This correction was performed by subtracting a low-pass filtered version of each image to the raw image, where the low-pass image is obtained by a mean filter (kernel of 50 pixels)[17]. Following uneven illumination correction, a histogram stretching is realized to enhance image contrast by saturating the $0.1 \%$ brightest and darkest pixels. Raw images are recorded without any pre-processing to allow accurate off-line processing described below.

\section{E. Off-line Image processing}

A flow diagram of our retinal image processing sequence is shown in Fig.3. Following the acquisition, each raw image was corrected for uneven background illumination as described above. The image quality of $\mathrm{AO}$-corrected image sequences varies over time, especially before and during blinks, and during fast and large fixational eye movements such as micro-saccades [18]. The spatial variance was used as a reliable image quality metric to select the best images of the sequence [17]. The final selected images were registered using a pairwise maximum likelihood sub-pixel approach [19]. We generated an average image on which we perform a histogram stretching to enhance contrast. For some cases, to improve the optical sectioning, we also performed deconvolution [20].

\section{RESULTS}

\section{A. Wavefront control internal performance}

The overall system performance can be assessed and optimized thanks to the calibration source (see Fig.1). In this configuration, the wavefront sensor beacon source is switched off and the calibration source, simulating an unresolved point source on the

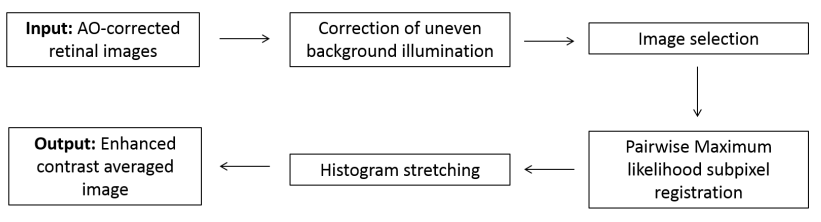

Fig. 3. General flow diagram of retinal image processing

retina, is switched on. In this scenario, only the aberrations introduced by the optics themselves are seen and corrected by the AO loop. This calibration source thus allows us to estimate the ultimate performance of the AO loop, i.e. without external aberrations. This parameter is usually called internal performance. The internal performance is evaluated by two criteria: Point Spread Function (from which the Strehl Ratio can be derived) and Rejection Transfer Function (from which a correction cut-off frequency can be derived).

The resulting Point Spread Function (PSF) is observed on the imaging camera with the Adaptive Optics loop closed on the calibration source. In this configuration the pupil is limited by the deformable mirror diaphragm $(5 \mathrm{~mm}$ diameter at the eye pupil plane). The typical internal PSF on the calibration source with AO correction is displayed in Figure 4. Strehl Ratio can then be derived from the PSF [21]. We have evaluated a Strehl Ratio superior to $90 \%$, demonstrating an excellent correction of the static aberrations, including non-common path aberrations.

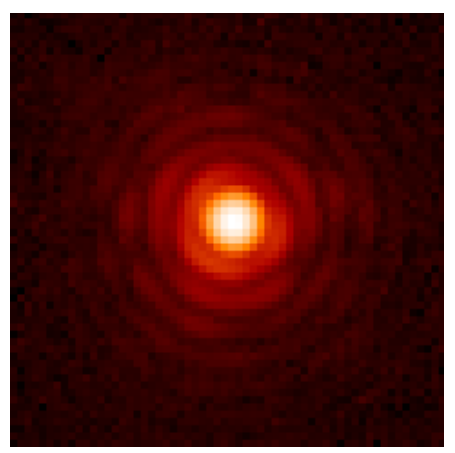

Fig. 4. Point Spread Function in closed loop on the calibration source $(\lambda=860 \mathrm{~nm})$. The color scale is logarithmic so as to reveal the ripples.

To evaluate the temporal performance of the AO loop, we consider the AO loop Rejection Transfer Function, which describes how different temporal frequencies of the aberrations are attenuated by the AO loop. Assuming a high signal to noise ratio on the calibration source, the rejection transfer function is the ratio between the residual phase aberrations temporal power spectrum density $S\left(\varphi^{\text {res }}\right)$ and the input phase aberrations temporal power spectrum density $S(\varphi)$ :

$$
\mathrm{RTF}=S\left(\varphi^{\mathrm{res}}\right) / S(\varphi)
$$

A standard way to measure this rejection transfer function is to inject known aberrations using the deformable mirror itself to introduce perturbations. A pink noise with a power spectrum density following a $f^{-1}$ law is generated and applied to each actuator of the deformable mirror (this type of colored noise avoids applying too much energy at high temporal frequencies that could degrade the deformable mirror). Wavefront measurements are recorded with the adaptive optics loop off (i.e. with 
input phase aberrations) and then with the adaptive optics loop on (i.e. with residual phase aberrations). For each slope measurement, a residual transfer function is obtained. These residual transfer functions are averaged to obtain one overall rejection transfer function, which is shown in Figure 5. This experimental rejection transfer function is compared to the theoretical one assuming a 2-frame delay and a 0.5 gain (which is the actual setting used during the acquisition). The good match demonstrates that no additional delay or jitter was present during the acquisition, and that the temporal performance of the loop is as expected. It has to be noted that even with a fast adaptive optics loop $(60 \mathrm{~Hz}$ in this case) and with a high gain (0.5), the loop cut-off frequency, i.e. the highest corrected temporal frequency (associated with $0 \mathrm{~dB}$ value) is approximately $4 \mathrm{~Hz}$.

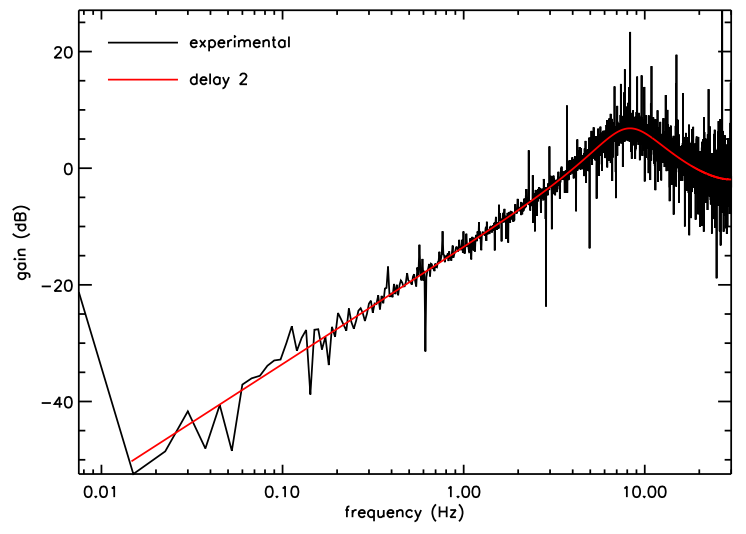

Fig. 5. Rejection Transfer function. Black: experimental rejection. Red: theoretical rejection with a 2-frame delay loop.

\section{B. Projection of high resolution features onto the retina}

Projecting high-resolution features onto the retina is required to benefit from the improved resolution and optical sectioning yielded by structured illumination microscopy. In our set-up, the illumination source is placed upstream of the deformable mirror, so benefiting from $\mathrm{AO}$-correction on the way into the retina.

To assess the capability to project and image high resolution features in the retina, we first project a point source pattern using the wavefront sensor beacon, with and without forward aberration compensation by the AO loop, in a real eye (see Figure 6). A better confinement of the laser beacon is noted with an $\mathrm{AO}$ correction, although multiply-scattered light makes a reliable size assessment difficult.

We next used a modified field stop on the imaging source, corresponding to an illumination pattern made of two half moons (i.e. a circular field diaphragm, with a cylindrical mask placed vertically in its center). Figure 7 presents the averaged image from a video sequence of the large illumination patch on a phantom eye (OCULAR Model OEMI-7, presenting 3.5 diopters myopia) with and without AO-correction. With AO-correction (on the right), the edges of the illumination patch can be easily identified. On the other hand, without AO-correction (on the left), the field diaphragm is almost imperceptible and no edges can be identified.

\section{Imaging Performance}

A standard way to assess retinal imaging performance is to observe the densely packed mosaic of photoreceptors. In order to

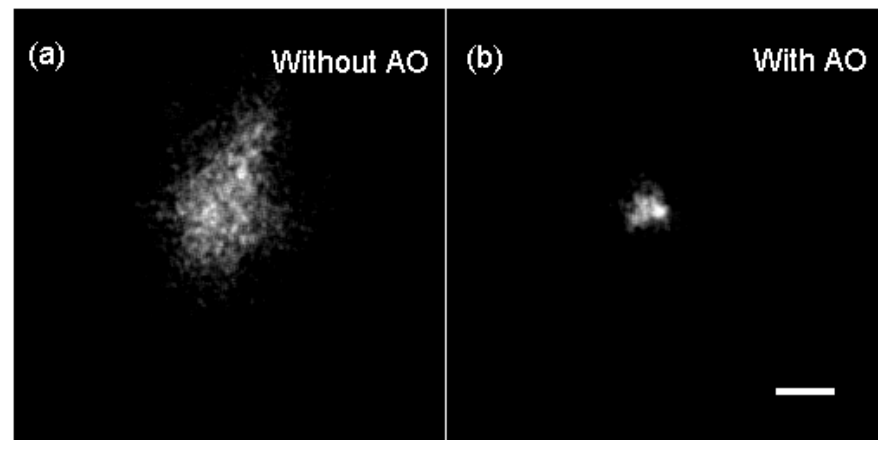

Fig. 6. Image of the wavefront sensor laser beacon at Subject EG's fovea (a) without Adaptive Optics correction and (b) with Adaptive Optics correction (in addition to the sharp dot corresponding to the laser beacon, some multiply-scattered light is present). Scale bar is $50 \mu \mathrm{m}$.
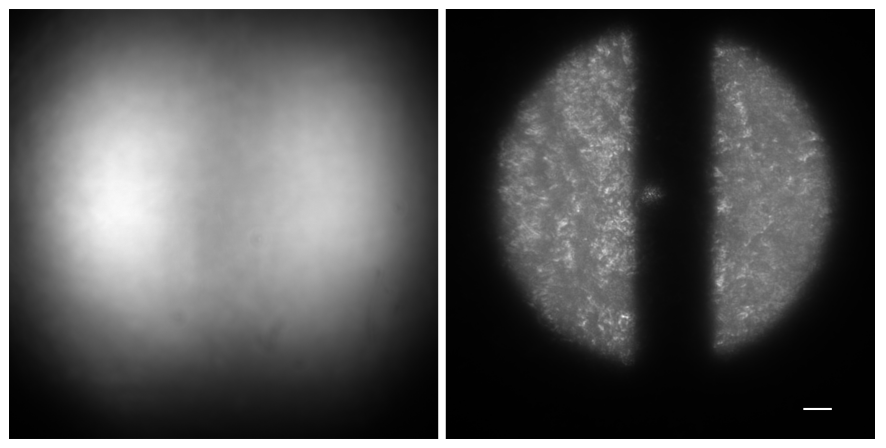

Fig. 7. Average image from a video sequence of the large illumination beam on a phantom eye. (Left) without AO correction. (Right) with AO correction. Scale bar is $50 \mu \mathrm{m}$.

illustrate the various steps in retinal image processing (see Fig.3) and to emphasize the gain of using a $50 \mathrm{~Hz}$ AO-loop correction, Figure 8 presents images of the same cone mosaic and the associated power spectral density with increasing quality: single raw image with a $10 \mathrm{~Hz}$ AO-loop correction $(\mathrm{a}, \mathrm{b})$, montaged images (made from 160 short exposure images) with uneven background correction with a $10 \mathrm{~Hz}$ AO-loop correction (c,d) and with a $50 \mathrm{~Hz}$ AO-loop correction (e),(f). This last image is then deconvolved to obtain the mosaic presented in (g),(h). At each step, we can observe in the power spectral density (PSD) maps that the typical Yellott's ring [22] becomes more highly contrasted at each step.

Further analysis can be done by plotting the radial average of the power spectral density from each of these images (Fig.8 (i)). Black dashed line highlights the typical photoreceptor spatial frequency peak for this region ( $4^{\circ}$ temporal), around 100 cycles $/ \mathrm{mm}$. It is possible to distinguish the photoreceptor spatial frequency peak in the raw image PSD (black line), but it is still dominated by noise. The signal-to-noise ratio at this frequency is dramatically improved by averaging 160 registered images (which represents $0.8 \mathrm{~s}$ image acquisition) and by correcting uneven background illumination (magenta and green lines). A clear signal-to-noise ratio gain at photoreceptors spatial frequency peak is witnessed between a $10 \mathrm{~Hz}$ (magenta) and a $50 \mathrm{~Hz}$ AO-loop correction (green). The frequency content of the averaged images almost reach diffraction limit, i.e. the optical cut-off frequency of the system at 323 cycles $/ \mathrm{mm}$ (blue dashed 
line), allowing an effective deconvolution yielding an improved image contrast.
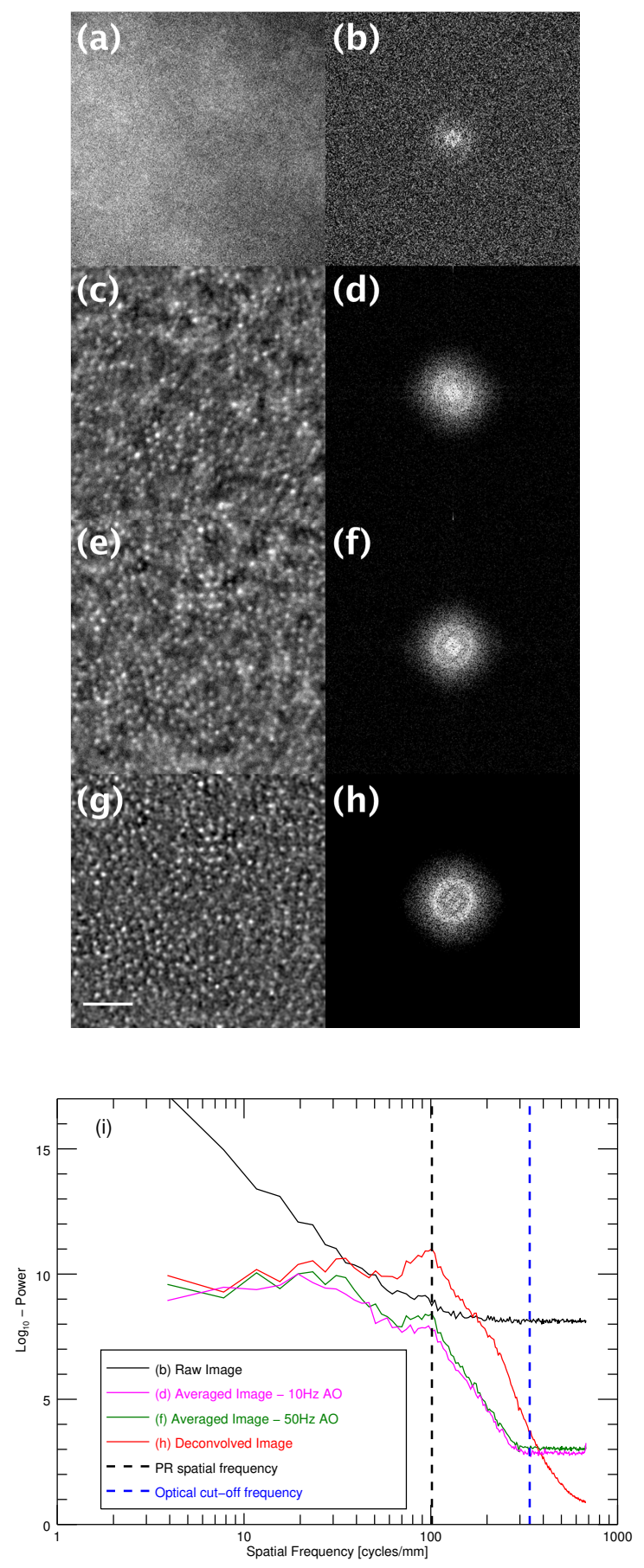

Fig. 8. Cone photoreceptor mosaic at $4^{\circ}$ temporal (subject EG). (a) Raw image and (b) its power spectral density (PSD) with a $10 \mathrm{~Hz}$ AO-loop correction. Corresponding 160 images average (c), after registration and correction of uneven background illumination and associated PSD (d). (e,f): same as (c,d) but with a $50 \mathrm{~Hz}$ AO-loop correction. (g,h): Image and associated PSD obtained after deconvolution of image (e). Photoreceptors images are represented in linear scale and PSD images represented in logarithm scale. Scale bar is $50 \mu \mathrm{m}$.(i) Radial average of the $\log 10$-power spectrum densities.

\section{Imaging various zones in the retina}

This AO-FIO system is able to resolve microscopic features as it has been shown previously. In this section, we illustrate the image quality yielded by the resolution and contrast of the instrument on various zones of the retina.

Figure 9 shows representative images of retinal vessels, ranging from $3 \mu \mathrm{m}$ capillaries to $80 \mu \mathrm{m}$ arteries. The resolution performance and the wide accessible field of view achieved by our platform are particularly suited to image the retinal vascular network, from small vessel structures - vessel walls (see red arrowheads in Figs. 9.a and 9.d) and capillaries (see yellow arrowheads in Fig. 9.c) - to large vessel structures - arterioveinous crossings or bifurcations (see Figs. 9.a and 9.b). The retinal nerve fiber layer is highly contrasted (see Figs. 9.b and 9.d). Such distortionless images of the retinal nerve fiber layer could be valuable to understand and recognize early cases of glaucoma [23]. These images also reveal how nerve fibers and vessels are intertwined: some vessels which are embedded in the nerve fiber layer (yellow arrowhead in Fig. 9.b) and red arrowhead in Fig. 9.d) and others are partially sitting on top the fiber layer (red arrowhead in Fig. 9.b) and yellow arrowhead in Fig. 9.d).

The combination of a large field of view, distortionless and high spatial resolution with the high temporal resolution of the system allows the visualization of large vessel deformations. An example is presented in Visualization $\mathbf{1}$ corresponding to the image sequence from which Fig.10 has been averaged (the blur on the vessel bend is actually due to its motion during the sequence). In this video we observe a $50 \mu \mathrm{m}$ diameter vessel, curved with a large bend radius, undergoing a horizontal deformation with the cardiac pulse.

Finally, a high-resolution image sequence of the Lamina Cribrosa was acquired resulting in the average image in Fig.11. We are able to resolve collagen fibers from various layers of the mesh-like structure (the yellow arrowhead shows a fiber inside a pore of the Lamina Cribrosa) and capillaries irrigating the Lamina Cribrosa (red arrowheads indicate a vessel crossing the laminar mesh). Blood flow in this capillaries is visible in the video sequence (see Visualization 2, the video corresponding to Fig.11).

\section{DISCUSSION}

This paper shows the capabilities of a high-performance adaptive optics loop coupled to a flood illumination retinal imager. Imperfect knowledge of the system (e.g. interaction matrix miscalibration, real-time computer jitter, deformable mirror oscillations) can be accommodated by the use of a smaller gain, but at the price of a reduced temporal bandwidth correction. The experimental adaptive optics rejection transfer function shown in section 3.A proves that our loop is stable at $60 \mathrm{~Hz}$ (with 2 frame delay, without jitter) with 0.5 loop gain, which is the maximum gain ensuring usual stability margins.

The benefit of a fast AO loop ( $50 \mathrm{~Hz}$ compared to $10 \mathrm{~Hz})$ is demonstrated in Fig. 8. The spectral content of the images reaches the $2.3 \mu \mathrm{m}$ diffraction limit (optical cut-off frequency) and the signal-to-noise of photoreceptors spatial frequency is improved by a factor of 4 at $50 \mathrm{~Hz}$ compared to $10 \mathrm{~Hz}$. Vessel wall structures, capillaries, and Lamina Cribrosa fibrinogen mesh are revealed on $3^{\circ} \times 2^{\circ}$ video sequences acquired at $200 \mathrm{~Hz}$. This high spatial resolution capability at a high frame rate over a large field of view is undoubtedly under-utilized in the retinal imaging community (apart from the commercial apparatus by 


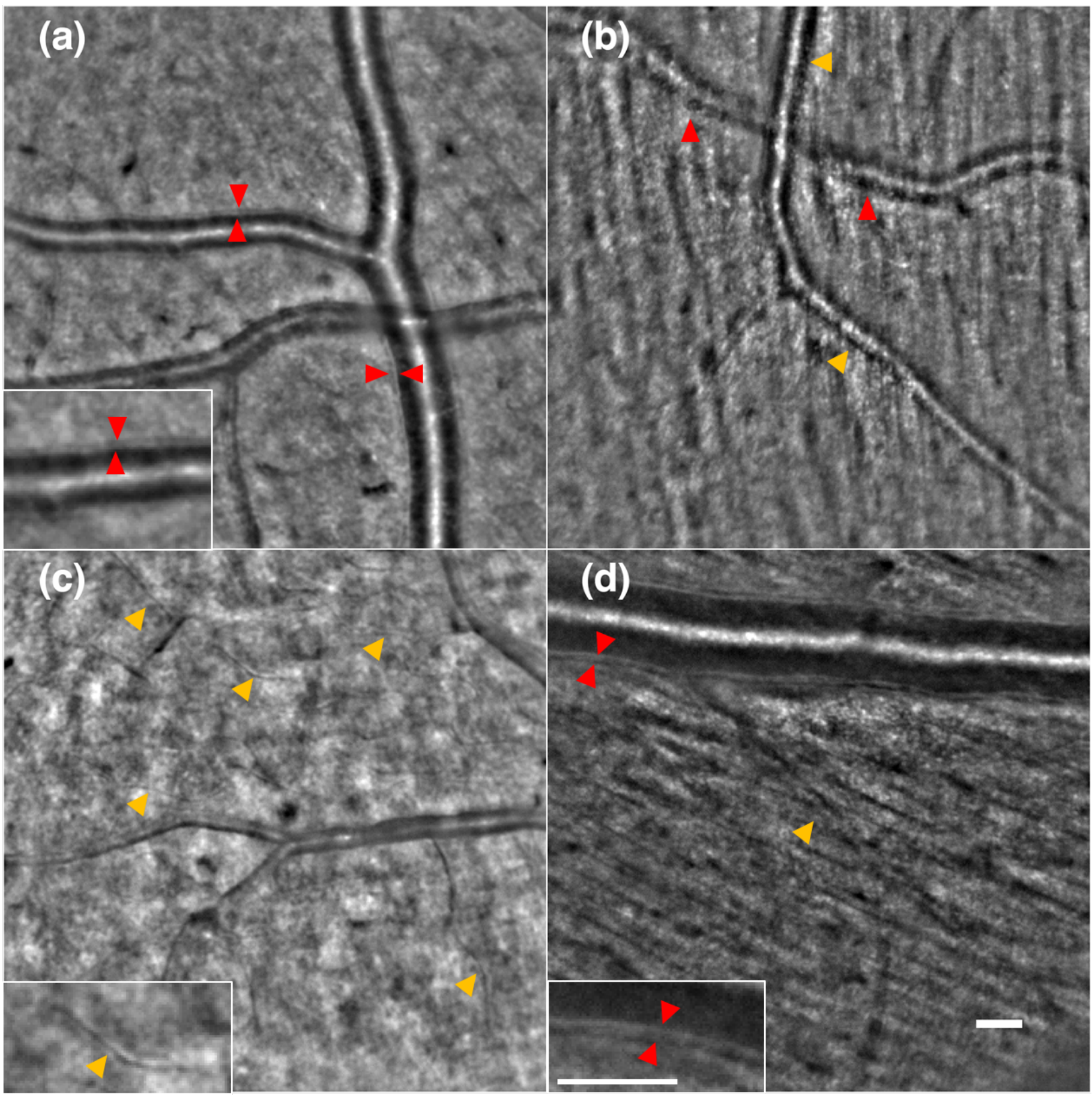

Fig. 9. (a)Average image of a bifurcated artery crossing a vein. (b)Average image of vessels crossing embedded in the retinal nerve fiber layer. One of the vessels is above the fiber layer (yellow arrowheads), the other one (red arrowhead) is below the fiber layer. (c) Average image of capillaries (yellow arrowheads) irrigating the retina.(d) Average image of retinal vessels, a $80 \mu \mathrm{m}$ retinal artery. Red arrowheads indicate vessel wall (thickness around $10 \mu \mathrm{m}$ ). Scale bar is $50 \mu \mathrm{m}$. 


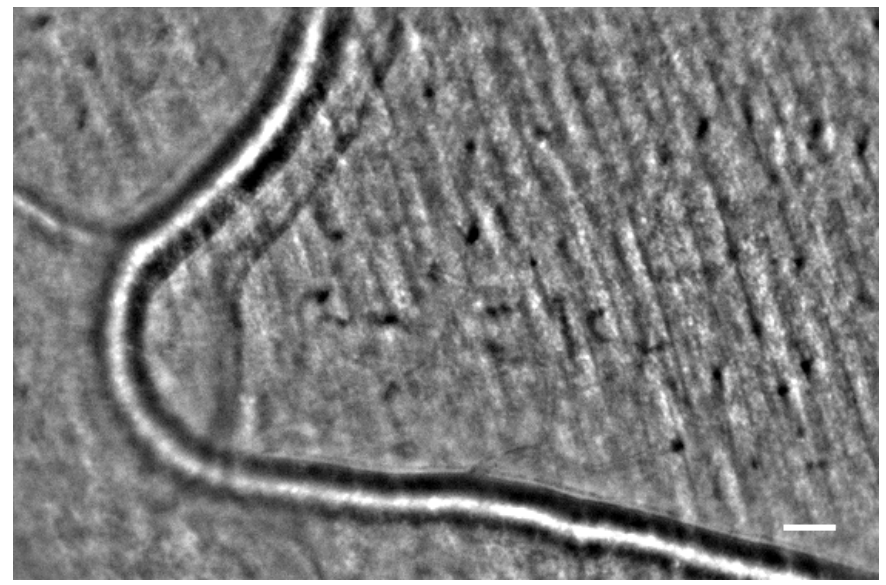

Fig. 10. Average image of a large curved vessel. In the video sequence (see Visualization 1) the vessel is deformed following cardiac pulses. Scale bar is $50 \mu \mathrm{m}$.
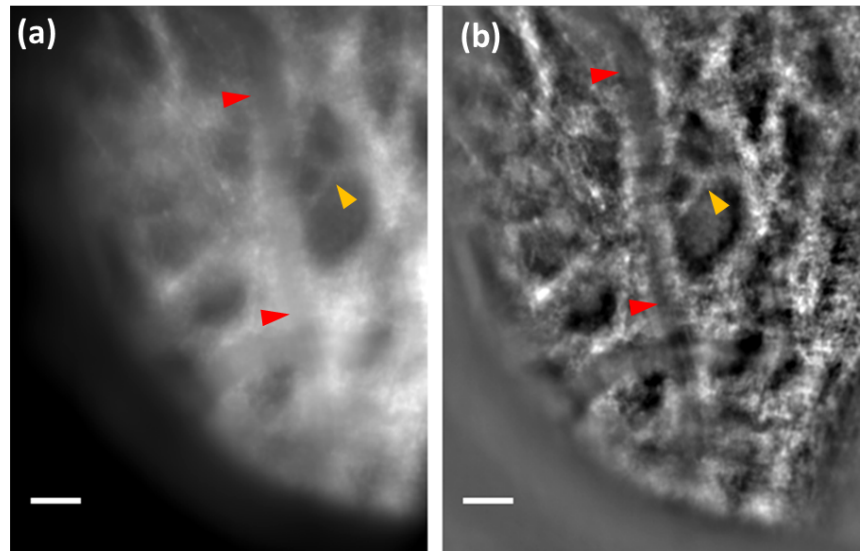

Fig. 11. Processed image of the Lamina Cribrosa before (a) and after (b) uneven illumination correction. Red arrowheads indicate a vessel inside the Lamina Cribrosa (blood flow is visible in the corresponding sequence, see Visualization 2). Yellow arrowhead points out fibrous branches from deeper layers of the Lamina Cribrosa. Scale bar is $50 \mu \mathrm{m}$.

Imagine Eyes), probably to an imperfect correction of the aberrations. To our knowledge, flood illumination systems have only been used with slower adaptive optics loops (typically $10 \mathrm{~Hz}$ ) and lower gains. Also, the importance of a correct conjugation of the eye pupil with the deformable mirror, a key aspect for anisoplanatism mitigation, is probably underestimated.

This is all the more important seeing that flood illumination modality has lower optical sectioning than the scanning laser ophthalmoscope. The only way to beat the photon noise entailed by defocused layers is to have high contrast features at medium to high spatial frequencies. The signal to noise ratio at these spatial frequencies is directly linked to adaptive optics performance. More generally, flood illumination Point Spread Function is the square root of confocal scanning laser ophthalmoscope Point Spread Function[24], making aberrations all the more detrimental, and a proper adaptive optics correction all the more critical.

The results presented here suggest many investigations to be pursued on our Adaptive-optics flood illumination ophthalmo- scope. For instance, we demonstrate in [25] that a fast adaptive optics loop enable a better capacity to separate layers in a flood illumination ophthalmoscope. The wide-field $200 \mathrm{~Hz}$ imaging capability could be an extremely valuable tool to assess red blood cell trajectories in vessels from small capillaries to large arteries or veins. Angiography at $593 \mathrm{~nm}$ in AO-FIO has been performed by Metha et al. [26], but the capability to obtain similar results at a wavelength that is comfortable for the patient and neutral in terms of visual stimulation (e.g. the $860 \mathrm{~nm}$ imaging wavelength used in the present paper) would be an asset for neurovascular coupling studies in the human eye. The dynamic study of vessel deformation under the systolic wave from video sequences such as in Visualization $\mathbf{1}$ could be used for local vascular elasticity assessment. Blood flow in capillaries at the surface of the Lamina Cribrosa (see Visualization 2), as well as distortionless nerve fiber layer high contrast images could be used in glaucoma early management. Last but not least, the double-pass correction of aberrations enables to project high spatial frequency patterns onto the retina. This paves the way towards structured illumination ophthalmoscopy, a technique that could reach AO-SLO optical sectioning and resolution with an AO-FIO setup.

The resolution performance achieved by our setup, thanks to careful control of $\mathrm{AO}$ parameters, opens up the prospect of a return to favor of flood illumination adaptive optics systems, particularly in view if their inherent advantages over scanning systems of distortionless, high-speed imaging.

\section{FUNDING}

Agence Nationale de la Recherche under grants CLOVIS3D (grant number ANR-14-CE17-0011), RHU LIGHT4DEAF (grant number ANR-15-RHUS-0001) and ERC Grant Agreement (grant number 610110).

\section{REFERENCES}

1. J. Liang, D. R. Williams, and D. T. Miller, "Supernormal vision and high-resolution retinal imaging through adaptive optics," JOSA A 14 2884-2892 (1997).

2. A. Roorda and D. R. Williams, "The arrangement of the three cone classes in the living human eye," Nature 397, 520 (1999).

3. A. Roorda, "Adaptive optics ophthalmoscopy," J. Refract. Surg. 16, S602-S607 (2000).

4. J.-F. Le Gargasson, M. Glanc, and P. Léna, "Retinal imaging with adaptive optics," Comptes Rendus de l'Acadèmie des Sci. IV-Physics 2, 1131-1138 (2001).

5. A. Roorda, F. Romero-Borja, W. J. Donnelly III, H. Queener, T. J. Hebert, and M. C. Campbell, "Adaptive optics scanning laser ophthalmoscopy," Opt. express 10, 405-412 (2002).

6. C. Viard, K. Nakashima, B. Lamory, M. Pâques, X. Levecq, and N. Château, "Imaging microscopic structures in pathological retinas using a flood-illumination adaptive optics retinal camera," (2011), vol. 7885, pp. 788509-788509-10.

7. M. Minsky, "Microscopy apparatus," (1961). US Patent 3,013,467.

8. A. Duan, P. A. Bedggood, B. V. Bui, and A. B. Metha, "Evidence of Flicker-Induced Functional Hyperaemia in the Smallest Vessels of the Human Retinal Blood Supply," PLOS ONE 11, e0162621 (2016).

9. A. Duan, P. A. Bedggood, A. B. Metha, and B. V. Bui, "Reactivity in the human retinal microvasculature measured during acute gas breathing provocations," Sci. Reports 7, 2113 (2017).

10. M. G. Gustafsson, "Surpassing the lateral resolution limit by a factor of two using structured illumination microscopy," J. microscopy 198 82-87 (2000).

11. M. A. Neil, R. Juškaitis, and T. Wilson, "Method of obtaining optical sectioning by using structured light in a conventional microscope," Opt. letters 22, 1905-1907 (1997). 
12. S. Gruppetta, "Structured illumination for in-vivo retinal imaging," in "Frontiers in Optics," (Optical Society of America, 2013), pp. FW2F-1.

13. J. Lu, B. Gu, X. Wang, and Y. Zhang, "High-speed adaptive optics line scan confocal retinal imaging for human eye," PloS one 12, e0169358 (2017).

14. J. Jarosz, P. Mecê, J.-M. Conan, C. Petit, M. Paques, and S. Meimon, "High temporal resolution aberrometry in a 50 -eye population and implications for adaptive optics error budget," Biomed. Opt. Express 8, 2088-2105 (2017).

15. S. Meimon, J. Jarosz, C. Petit, E. G. Salas, K. Grieve, J.-M. Conan, B. Emica, M. Paques, and K. Irsch, "Pupil motion analysis and tracking in ophthalmic systems equipped with wavefront sensing technology," Appl. Opt. 56, D66-D71 (2017).

16. F. Roddier, "Adaptive optics in astronomy," (1999).

17. G. Ramaswamy and N. Devaney, "Pre-processing, registration and selection of adaptive optics corrected retinal images," Ophthalmic Physiol. Opt. 33, 527-539 (2013).

18. P. Mecê, J. Jarosz, J.-M. Conan, C. Petit, K. Grieve, M. Paques, and S. Meimon, "Fixational eye movement: a negligible source of dynamic aberration," Biomed. Opt. Express 9, 717-727 (2018).

19. D. Gratadour, L. M. Mugnier, and D. Rouan, "Sub-pixel image registration with a maximum likelihood estimator. Application to the first adaptive optics observations of Arp 220 in the L' band," Astron. Astrophys. 443, 357-365 (2005).

20. L. Blanco and L. Mugnier, "Marginal blind deconvolution of adaptive optics retinal images," Opt. express 19, 23227-23239 (2011).

21. J.-F. Sauvage, T. Fusco, M. Lamb, J. Girard, M. Brinkmann, A. Guesalaga, P. Wizinowich, J. O'Neal, M. N'Diaye, A. Vigan et al., "Tackling down the low wind effect on sphere instrument," in "Adaptive Optics Systems V," , vol. 9909 (International Society for Optics and Photonics, 2016), vol. 9909, p. 990916.

22. R. F. Cooper, C. S. Langlo, A. Dubra, and J. Carroll, "Automatic detection of modal spacing (Yellott's ring) in adaptive optics scanning light ophthalmoscope images," Ophthalmic Physiol. Opt. 33, 540-549 (2013).

23. G. Ramaswamy, M. Lombardo, and N. Devaney, "Registration of adaptive optics corrected retinal nerve fiber layer (RNFL) images," Biomed. Opt. Express 5, 1941-1951 (2014).

24. K. Venkateswaran, A. Roorda, and F. Romero-Borja, "Theoretical modeling and evaluation of the axial resolution of the adaptive optics scanning laser ophthalmoscope," J. biomedical optics 9, 132-139 (2004).

25. P. Mecê, C. Petit, E. Gofas-Salas, K. Grieve, M. Paques, and S. Meimon, "Enhancing axial resolution in nonconfocal adaptive-optics ophthalmoscopes," Biomed. Opt. Express (submitted) (2018).

26. P. Bedggood and A. Metha, "Direct visualization and characterization of erythrocyte flow in human retinal capillaries," Biomed. optics express 3, 3264-3277 (2012). 